\title{
Reconstruction and display of curvilinear objects from optical section data using 3-D curve fitting algorithms
}

\author{
E. SHELDEN* \& D. A. KNECHT $\dagger$ \\ *Department of Anatomy and Cell Biology, University of Michigan Medical School, Ann Arbor, MI \\ 48109-0616, U.S.A. \\ †Department of Molecular and Cell Biology, University of Connecticut, Storrs, CT 06269, U.S.A.
}

Key words. 3-D reconstruction, confocal microscopy, cytoskeleton, microtubules.

\begin{abstract}
Summary
Biological objects resembling filaments are often highly elongated while presenting a small cross-sectional area. Examination of such objects requires acquisition of images from regions large enough to contain entire objects, but at sufficiently high resolution to resolve individual filaments. These requirements complicate the application of conventional optical sectioning and volume reconstruction techniques. For example, objective lenses used to acquire images of entire filaments or filament networks may lack sufficient depth (Z) resolution to localize filament cross-sections along the optical axis. Because volume reconstruction techniques consider only the information represented by a single volume element (voxel), views of filament networks reconstructed from images obtained at low Z-resolution will not accurately represent filament morphology. A possible solution to these problems is to simultaneously utilize all available information on the path of an object by fitting 3-D curves through data points localized in 2-D images. Here, we present an application of this approach to the reconstruction of microtubule networks from 2-D optical sections obtained using confocal microscopy, and to synthesized curves which have been distorted using a simple mathematical model of optical sectioning artefacts. Our results demonstrate that this strategy can produce high resolution 3-D views of filamentous objects from a small number of optical sections.
\end{abstract}

\section{Introduction}

A variety of biomedical research questions require visualization and analysis of structures resembling 3-D curvilinear objects (filaments). Examples of such structures include nerves, blood vessels and cytoskeletal filaments as well as the path of cell movements during normal development, cancer cell metastasis and the immune response. The development of optical sectioning techniques such as Correspondence to: Eric Shelden. confocal microscopy, an expanding spectrum of fluorescent probes and inexpensive and powerful image processing workstations have generated renewed interest in the visualization of 3-D data obtained from examination of biological structures. Strategies for the analysis and display of globular objects from optical sections have been aggressively developed and include voxel-based approaches in which image pixels in digitized 2-D optical sections are treated as unique 3-D data points (e.g. Kajiya \& Von Herzen, 1984; Frieder et al., 1985), and object-based approaches where local object surface regions are first detected and then modelled with polygon meshworks (e.g. Lorensen \& Cline, 1987; Guilak, 1994). However, these approaches consider only individual voxels or regions defined by a voxel and its immediate neighbours when generating rendered views of 3-D data sets. As a result, these approaches require that image resolution in both the lateral $(X Y)$ and vertical (Z) dimensions be sufficiently high to represent the smallest object of interest, often requiring acquisition and processing of large volumetric data sets. In cases where the resolution of acquired images is low relative to the size of the object(s) being examined, such voxel-based approaches produce views of objects which are distorted and unnatural in appearance. Because the resolution of a microscope is lower along the optical axis (the $\mathrm{Z}$ dimension) than in the lateral (XY) plane (see discussion by Taylor \& Salmon, 1989; Cox \& Sheppard, 1993) such artefacts are particularly apparent when viewing rendered images of optically sectioned volumes from angles other than along the original optical axis.

Because curvilinear objects may be orders of magnitude larger in length than thickness, they present unique challenges to optical sectioning and volume visualization techniques. For example, a $10 \times 0 \cdot 25 \mathrm{NA}$ non-immersion objective with a calculated axial resolution of $7.9 \mu \mathrm{m}$ available in our imaging facility might be used to visualize the overall shape of neurite outgrowths extending over several hundred micrometres, but cannot satisfactorily 
determine the $z$-dimensional location of nerve fibres less than $1 \mu \mathrm{m}$ in diameter. Analogous problems are encountered in the visualization of the 3-D distribution of subcellular cytoskeletal filaments or extracellular matrix molecules. For example, high-NA objective lenses with depth discrimination approaching resolution limits along the optical axis are often used in the study of intracellular detail. However, the axial resolution of a $100 \times 1 \cdot 4$ NA oilimmersion objective is $\approx 0.3 \mu \mathrm{m}$ (Taylor \& Salmon, 1989) while the diameter of cytoskeletal elements, even when thickened by decoration with fluorescent antibodies, remains well below this limit. Rendered images of cytoskeletal networks therefore display sampling artefacts when viewed at angles other than along the optical axis (for example, see Results, below).

Other factors involved in image acquisition can further limit the effective axial resolution of microscope systems used to examine filamentous objects. For example, Wilson notes that the axial resolution of confocal imaging systems commonly used for optical sectioning is nearly twice as low for objects resembling lines than for those resembling a planar surface (Wilson, 1990). Additionally, in the study of living specimens, photobleaching, or the necessity of acquiring images of rapidly changing scenes and faintly fluorescent objects, may further limit the axial resolution of acquired images or the number of useful images which can be captured to represent a volume over a given period. These problems are particularly common to the use of laser scanning confocal systems where scanning mirrors and the confocal apertures reduce the brightness of the final image (Pawley, 1989). To increase image intensity, an enlarged pinhole aperture may be used, at some cost in axial resolution (Brakenhoff etal., 1989), or extended image integration times may be required to build up a useable image. As a result of these practical considerations, data obtained by optical sectioning techniques are often seriously undersampled in the $\mathrm{Z}$ dimension with respect to the thickness of objects being examined.

Various investigators have developed techniques which can increase both the lateral and $\mathrm{Z}$ dimensional resolution of optical section images. Some, such as deconvolution and other image restoration techniques, seek to enhance the axial resolution of microscope optical systems by first characterizing the effects of an optical system on light emanating from a point light source. The point-spread function can be used to computationally remove the contribution of light emanating from objects in neighbouring image planes from individual optical sections (for reviews see Agard et al., 1989; Shaw, 1993) or to localize small fluorescent objects more precisely than would otherwise be possible (for example, see Carrington etal., 1995). In cases where the morphology of the objects being examined can be approximated by curved lines, 3-D skeletonization routines have also been utilized to reduce the artefactual Z-dimensional thickening of objects produced by objective lenses with low Z-axis resolution (Cohen et al., 1994). However, these algorithms involve extensive (often iterative) calculation and are most effectively applied to large data sets obtained at high $\mathrm{Z}$ resolution, further increasing the computational power required to generate rendered images.

Modelling methods have also been used to visualize blood vessels and similar objects (Barillot et al., 1985; Fuji et al., 1986). These methods first approximate the contours of individual objects using a small number of data points and can rapidly generate views of objects from any angle. Modern computing methods allow such models to be rapidly constructed and viewed from any angle. However, previously applied modelling methods have relied on the approximation of object morphology with polygonal meshworks determined by analysis of individual of neighbouring voxels comprising optical section data sets, and this approach is therefore constrained by the same resolution problems inherent to volume rendering.

An alternative solution is to calculate an object's morphology based on prior knowledge of its likely shape. For example, in the case of simple linear objects, the determination of two end points in three dimensions would be sufficient to predict the location of all intervening points in the object. Users of computer-aided drawing programs will also recall that complex curves can be created by defining a small number of restraining points. Thus for more complex curvilinear objects it may be possible to predict an object's morphology from a limited set of data points. The current work presents a novel application of 3-D Bezier curve fitting algorithms to the visualization of curvilinear objects from optical section images, and the first application of such techniques to the visualization of cytoskeletal arrays. Specifically, the methods presented here first establish the relationship and linkage of 2-D linear segments located within optically sectioned images using software tools similar to those used in the analysis of chromosome morphology (for example, see Agard etal., 1989) described by Mathog (1985). Briefly, we have implemented a system in which serial optical sections can be displayed on a computer monitor in sequence, under user control. A mouse-driven cursor is used to select points along individual linear structures and to specify the order of line segments making up each object. The computer records the $X$ and $Y$ coordinates of selected points as well as the image number in which each selection is made.

In our hands, simple linear interpolation of the selected data points as described previously (Mathog, 1985) does not represent the paths of filamentous objects with sufficient accuracy to allow analysis of the distribution and morphology of many filaments in the same view (see Results). We have therefore implemented a 3-D Bezier curve-fitting algorithm to predict each object's morphology from the 
user-selected data points. We present an application of these methods to confocal images of microtubules obtained from fixed and immunostained Dictyostelium amoebae and to synthetic curvilinear objects which have been computationally sectioned and modified using a simple model of the distortion generated by the confocal microscope system. The results presented here reveal that these techniques can significantly improve the visualization and analysis of 3-D filamentous structures from optically sectioned volumes, and are particularly applicable to studies in which the organization and morphology of filament populations must be analysed.

\section{Methods}

\section{Cell culture and immunostaining}

Dictyostelium amoebae (strain NC4 A2) were cultured in $6 \mathrm{~cm}$ tissue culture dishes containing HL5 medium as described previously (Shelden \& Knecht, 1995), harvested by pipetting, and seeded onto untreated glass coverslips in $3.5 \mathrm{~cm}$ tissue culture dishes containing additional medium. Cells were allowed to attach overnight before further processing. For immunofluorescence observations, cells were simultaneously lysed and fixed using $2 \cdot 5 \%$ formaldehyde, $0 \cdot 1 \%$ glutaraldehyde, 0.01\% Triton-X 100 in buffer $\left(20 \mathrm{~mm} \mathrm{KH}_{2} \mathrm{PO}_{4}, 20 \mathrm{~mm}\right.$ $\mathrm{K}_{2} \mathrm{HPO}_{4}, 2$ mм $\mathrm{MgSO}_{4}$, 5 mм EGTA, 10 mм PIPES, pH 6.8) for $10 \mathrm{~min}$. Fixed cells were rinsed in PBS followed by an incubation in $1 \mathrm{mg} \mathrm{mL}^{-1}$ sodium borohydrate for $30 \mathrm{~min}$ to quench glutaraldehyde autofluorescence. Cells were then immunostained by incubation for $1 \mathrm{~h}$ with a 1:200 dilution of anti- $\alpha$ tubulin primary antibody (clone DM1 A, Sigma Chemical Co) and a second incubation with a 1:500 dilution of fluorescein-labelled antimouse IgG secondary antibodies (Sigma Chemical Co.). Cells were mounted in PBS containing $50 \%$ glycerol and $0 \cdot 1 \%$ phenylene diamine as an antifade agent using small pieces of modelling clay as spacers and the preparation was sealed with nail polish.

\section{Image acquisition and processing}

Imaging of fluorescent microtubule arrays was accomplished using a BioRad MRC600 laser scanning confocal microscope unit mounted on a Zeiss Axiovert 100 equipped with a $100 \times 1 \cdot 3 \mathrm{NA}$ oil-immersion objective. Z-series images were collected at $1 \mu \mathrm{m}$ intervals at the lowest magnification setting. Images were reduced in size by a factor of two before application of volume rendering or curve-fitting techniques, giving a final $X, Y, Z$ voxel size of $0 \cdot 32 \times 0 \cdot 32 \times 1 \cdot 0 \mu \mathrm{m}$.

Confocal images were first contrast stretched within NIHImage (NIH Image is a public domain image processing program written by Wayne Rasband at the U.S. National Institutes of Health and available from the Internet by anonymous FTP from zippy.nimh.nih.gov or on floppy disk from the National Technical Information Service, Springfield, Virginia, part number PB93-504868) and then rendered using a 3-D visualization program written by on of the authors (E.S.) running on a Macintosh computer (Apple PowerMac 7500/100). The program generates image projections at various angles through volumetric data sets using several algorithms. A 'semitransparent' pixel algorithm was used to generate all volume-rendered images presented here.

Bezier curve-fitted images were generated by loading Zseries images into the NIH-Image program. Individual microtubules were visually identified by paging up and down through the image series. Once identified, each microtubule was traced throughout the sequence of images containing the microtubule by tracing in sequence the microtubule segment contained within each sequential images. A 'macro' set of user defined commands was used to place the $x$ and $y$ coordinates of the start and end points of each line segment into a text-formatted data file along with the image number in the format (xstart,ystart,xend,yend,image). In addition, the macro marked each traced microtubule segment in red to prevent tracing the same segment for other microtubules. The termination of each microtubule trace was designated by writing a sequence of zeros to the data file in the format $(0,0,0,0,0)$.

Data generated in the manner described above were further manipulated using programs written in $\mathrm{C}$ using Metrowerks CodeWarrior running on a Power Macintosh (7500/100). Curve-fitting programs and NIH-Image macros are available free of charge from the authors. Because the extent of a microtubule in one image plane often overlaps the connected microtubule segment found in neighbouring planes, we chose to modify microtubule segment data by retaining only the 3-D coordinate of the midpoint of each traced line segment defined as described above. Coordinates of microtubule end-points were also retained and utilized to constrain the fitted curve through the original traced end-points. Line segments defined by the starting XYZ coordinate, intervening line segment midpoints, and end point coordinate were then used to generate Bezier curves using the algorithm published by Chasen (1975) and the XYZ coordinates of the 3-D fitted curves written in tab-delimited text format to a new data file. Finally, this data file was in turn read by a third program and the 3-D coordinates describing each microtubule converted to a standard DXF file description. The resulting DXF file was imported into a commercial rendering package (Strata Studio Pro, Strata Inc.) for final rendering.

\section{Synthesis of test objects and modelling of optical sectioning}

Synthetic curves were generated in NIH-Image using a mouse-driven cursor. We chose to model objects of a 


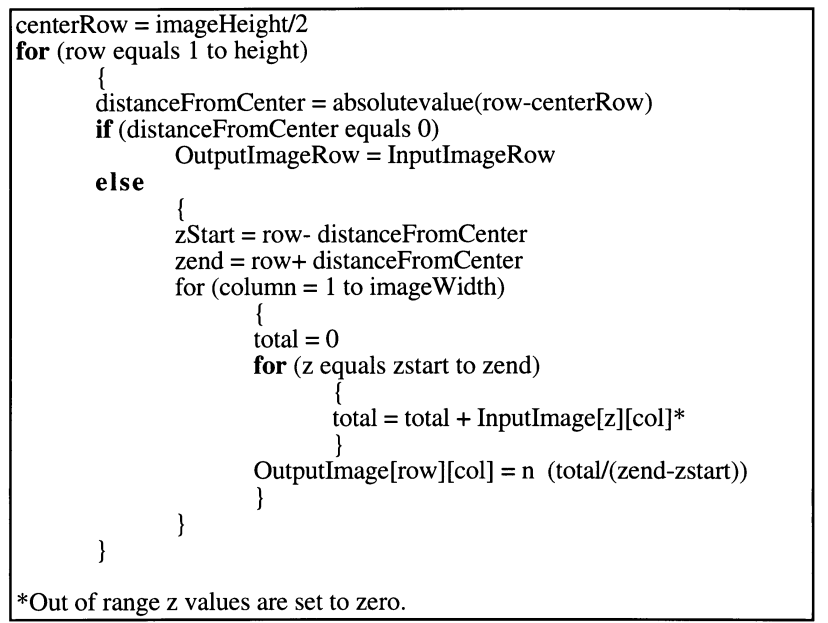

Fig. 1. Pseudocode description of the algorithm used to model the effects of the confocal point spread function on optical section images. Results most closely resembled those generated by the confocal microscope when $n=0 \cdot 2$.

dimension which suitably represented a diffraction-limited view of a microtubule. Images were created which were 250 pixels tall and 500 pixels wide. Curves were drawn using a 4-pixel-wide brush tool. We assumed a pixel/micrometre ratio of 20 , giving a line thickness of $0.2 \mu \mathrm{m}$, approximately the lateral resolution of the light microscope. Dimensions of each curve were chosen so as to model a microtubule originating at the centre and extending to the periphery of a cell $10 \mu \mathrm{m}$ tall and $40 \mu \mathrm{m}$ in diameter.

Each test image was divided into a vertical series of images using a moving window 25 pixels in height and 500 pixels wide. This window was scanned over the original image to generate a series of 'optical sections' representing $1 \cdot 2 \mu \mathrm{m}$ of vertical space. The window was moved only 20 pixels between images, thereby modelling a $10 \%$ contribution of out-of-focus information from each neighbouring image plane. Each 'section' was vertically blurred using an algorithm which assumes that the centre horizontal row of pixels is 'in focus' and experiences no blurring, while rows more distant from the centre experience increasing amounts of blur. The algorithm used is presented in pseudocode (Fig. 1). Each section was vertically projected using either a brightest-pixel or an average-pixel algorithm onto a single pixel plane, thus modelling the acquisition of a single image plane representing the $1 \cdot 2-\mu \mathrm{m}$-thick optical section. The two projection methods produced equivalent final results (not shown). Finally, the combined sequence of projected sections was used as input data for the reconstruction method described above.
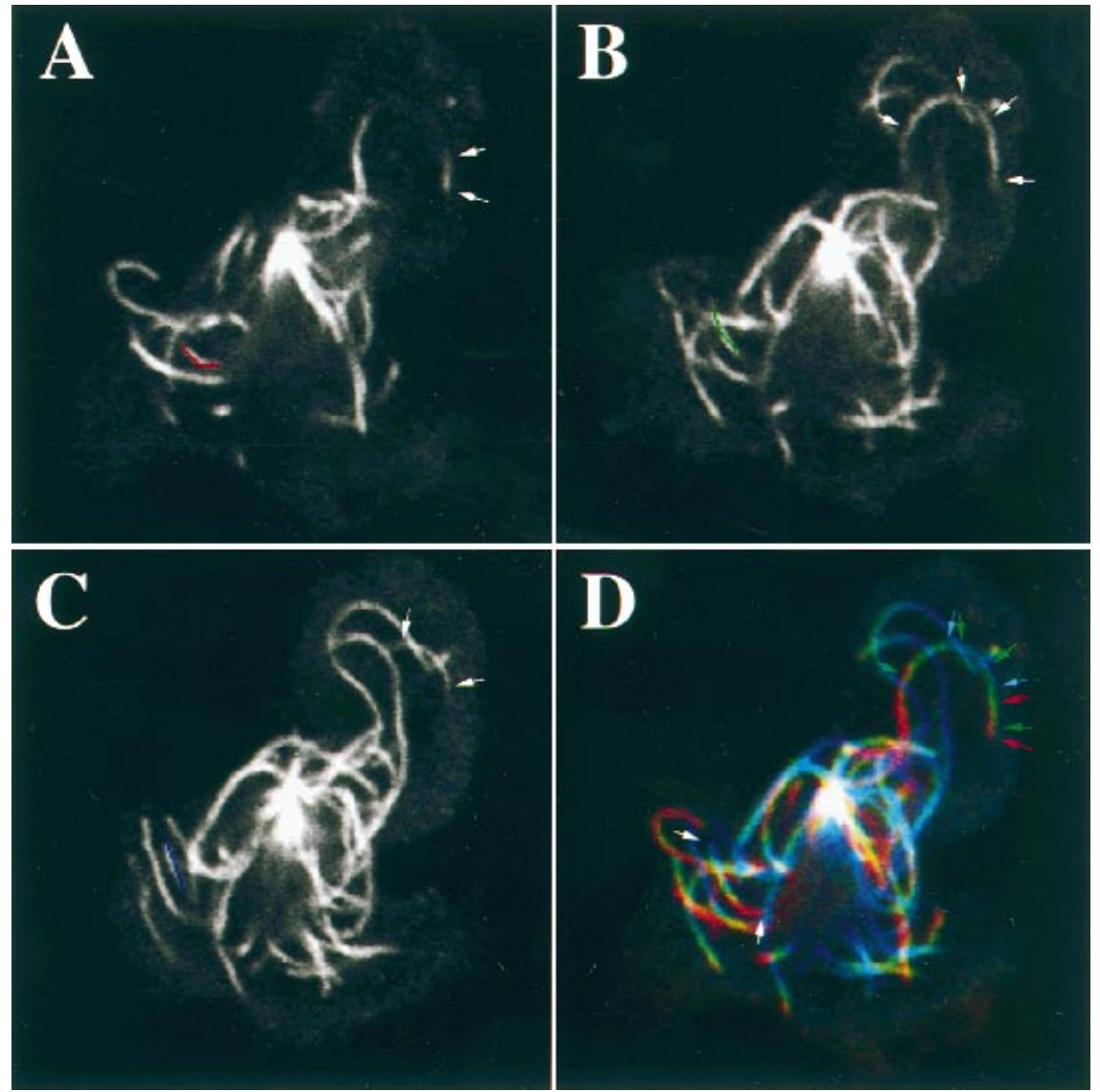

Fig. 2. Three adjacent confocal images of immunostained microtubules obtained at $1-\mu \mathrm{m}$ intervals. $(\mathrm{A}-\mathrm{C})$ The start and end points of traced microtubule segments for a single microtubule are shown in adjacent optical sections (arrows). Superimposition of the three segments reveals considerable overlap of information between adjacent images (coloured arrows, D). Also shown are colour-coded line segments generated during the tracing of a single microtubule $(\mathrm{A}-\mathrm{C})$. Images and arrows are colourcoded, with red indicating the highest plane of focus and blue indicating the plane of focus closest to the cell substrate. Width of field of view of each panel $=13 \mu \mathrm{m}$. 
Fig. 3. $X Z$ section images through a single microtubule. (A) An image obtained before depth correction reveals that individual optical sections do not fully represent the volume over which the data are collected. (B) Correction of the $\mathrm{Z}$ dimension by linear interpolation restores the volume to its original dimension but introduces artefactual elongation of the microtubule profile. (C) A hand-traced pathway through the image data gives an approximate view of what the microtubule should have looked like. Bar $2 \mu \mathrm{m}$.

\section{Results}

Adjacent confocal microscope images of cytoskeletal elements often contain out-of-focus information from neighbouring planes, particularly when the focal change between each image is smaller than the true Z-axis resolution of the optical system. For example, Fig. 2(A-C) shows adjacent images of fluorescently stained microtubules

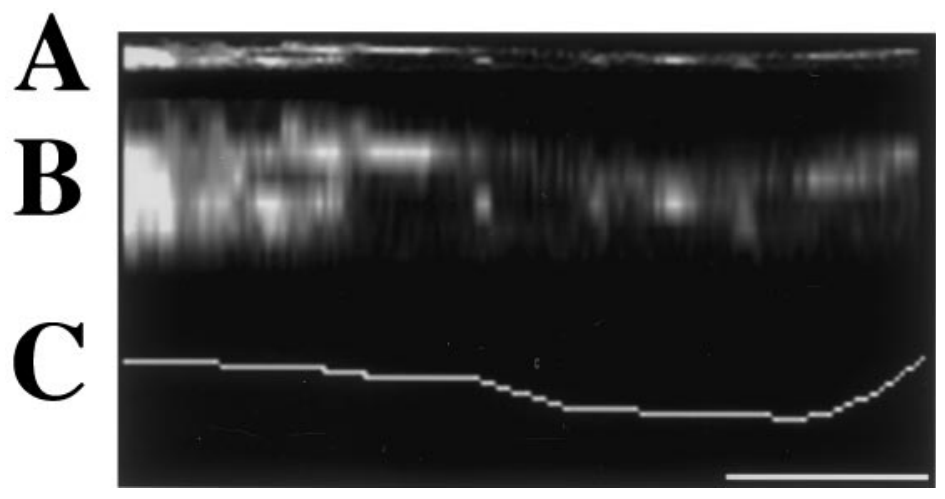

obtained using a step size of $\approx 1 \mu \mathrm{m}$. We judge these filaments to be individual microtubules on the basis of their similarity in conformation and fluorescence intensity distribution to fluorescently stained microtubule observed by both light and electron microscopy (Sammak \& Borisy, 1988) and to that of fluorescent microtubules observed in vivo (Shelden \& Wadsworth, 1993 and others cited in this previous publication). The length of individual microtubule

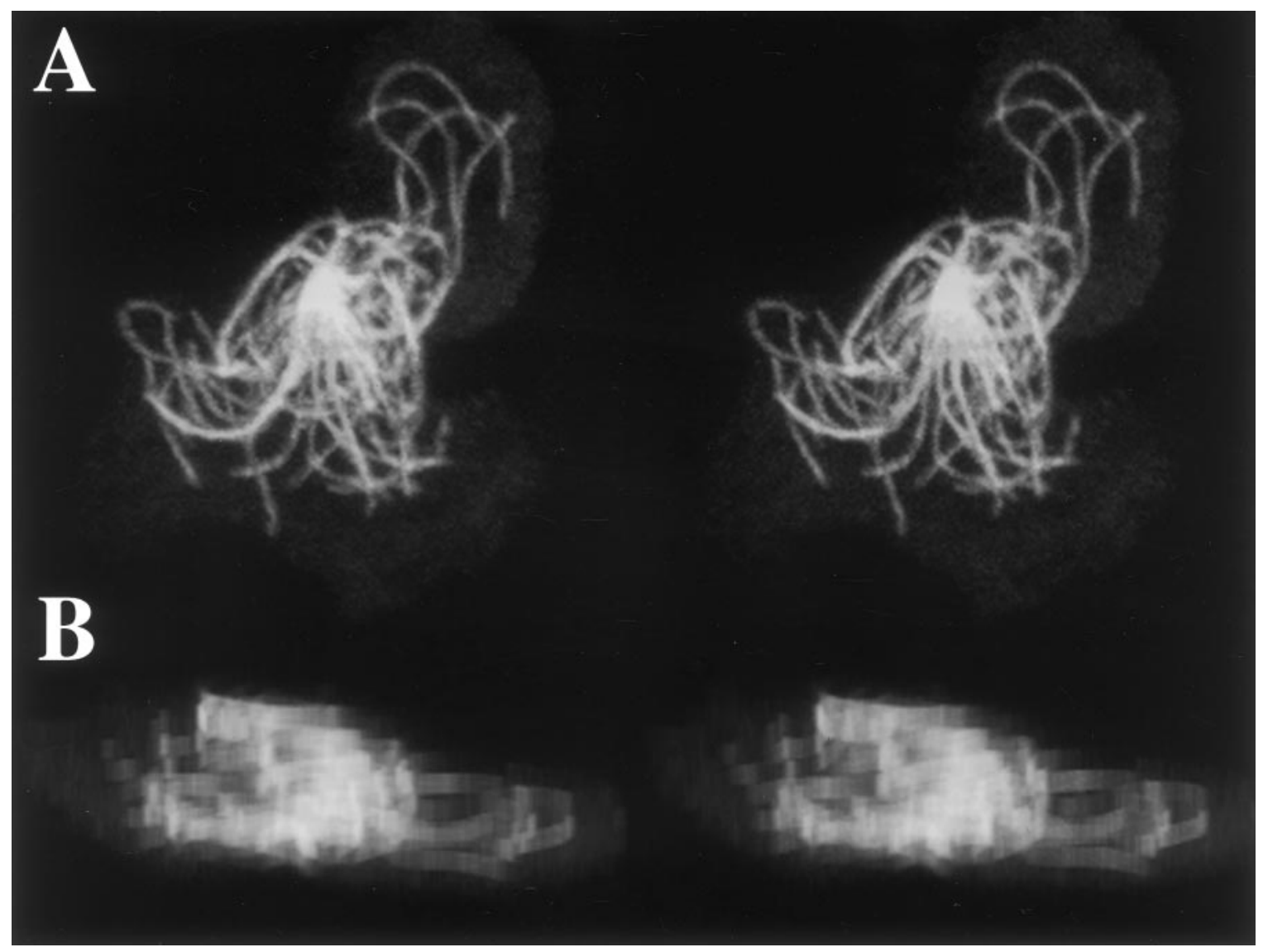

Fig. 4. Stereo images of confocal image data obtained by volume rendering techniques. (A) Images obtained by projection along or at $5^{\circ}$ to the optical axis of the microscope retain the original clarity and resolution of the individual optical sections. (B) When viewed after $90^{\circ}$ rotation on the optical $(Z)$ axis and $75^{\circ}$ along the $X$ axis, however, artefactual thickening of the microtubule segments degrades the image and obscures the spatial relationship of individual microtubules. Stereo pairs are arranged for the cross-eye viewing method. 
segments in each optical section is indicated by small arrows (Fig. 2A-C). In Fig. 2(D) the three images have been colour coded and combined. Overlap of the microtubule segments can be observed (examine the extent of colourcoded arrow pairs in Fig. 2) and will lead to distorted views of the object treated with volume rendering methods. The sequence of segment tracing can also be seen in the combined image (Fig. 2D). The distal end of the microtubule found in the upper image plane (colour-coded red) would be traced first. The segment in the adjacent lower plane (green) would be traced next, and then the segment found in the lowest image (blue). At this point the microtubule can be seen to curve upward again. Thus, the segment located in the middle image plane (green) would be traced next. The last traced segment is found closest to the cell centre and is again found in the uppermost image (red). Also shown are similarly colour-coded paths generated during the tracing of part of a single microtubule (Fig. 2A-C). The complete trace is comprises three line segments traversing the three optical sections (Fig. 2-D).

Figure 3 shows a vertical (XZ) section through the volumetric data set along the traced microtubule indicated in Fig. 2 both before (Fig. 3A) and after (Fig. 3B) Z-scaling to correct the Z-dimensional pixel $/ \mu \mathrm{m}$ ratio. Both overlap of line segments in adjacent images and thickening of the microtubule segments caused by $\mathrm{Z}$ scaling of image data contribute to distortion of the microtubule in the final image (Fig. 3B). Microtubules orientated normal to the image plane are seen in cross-section and appear as bright spots below the path of the longitudinally orientated microtubule.
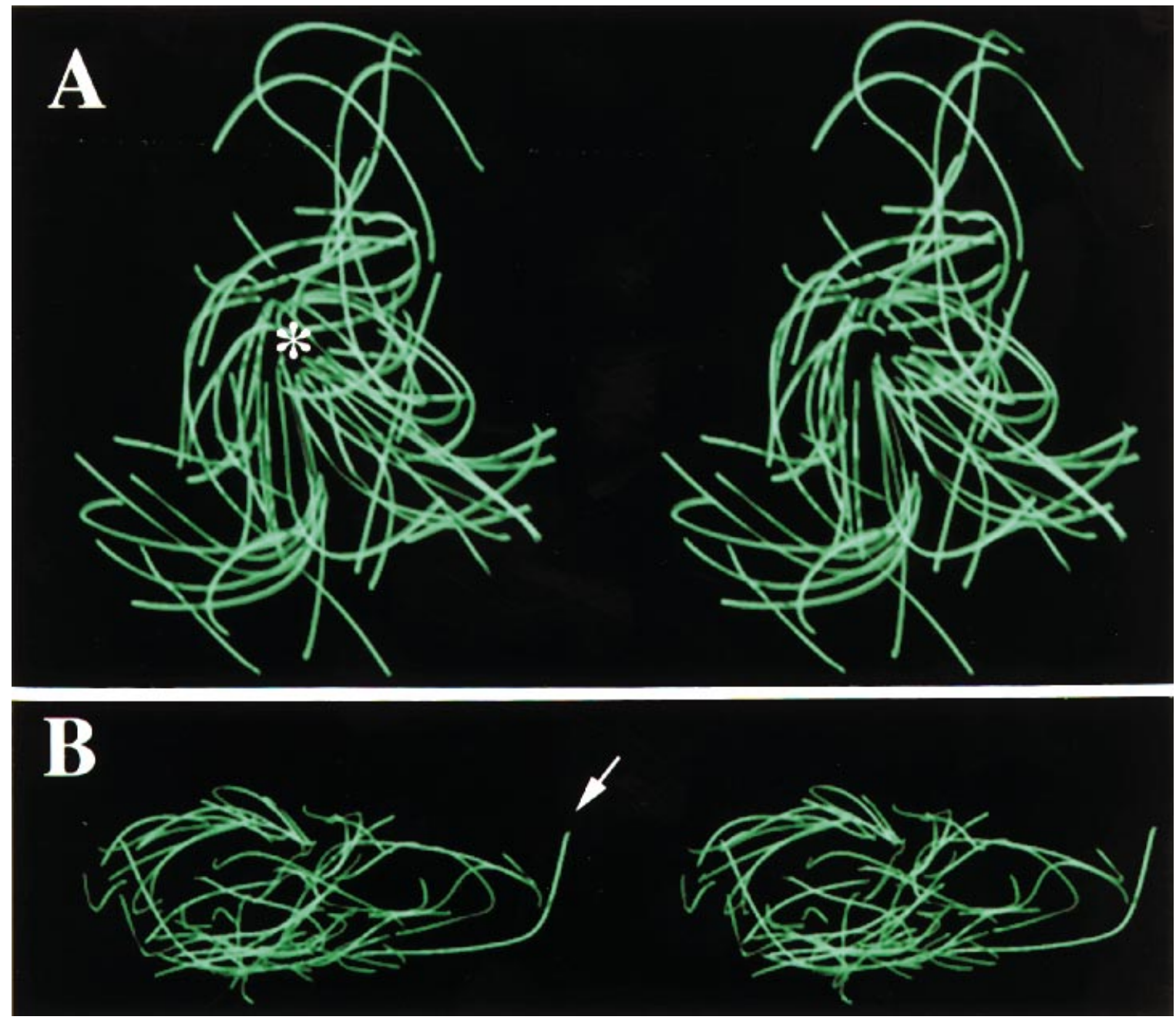

Fig. 5. Stereo images obtained by rendering of 3-D traced curve data. (A) Comparison of images obtained by viewing the data along or at $5^{\circ}$ to the original optical axis with volume rendered views (see Fig. 3A) suggests that curves fit through the line segment mid-point data adequately represent the paths of individual microtubules in three dimensions. (B) Views obtained after $90^{\circ}$ rotation along the optical axis and $75^{\circ}$ on the $X$ axis retain clarity even after depth correction and provide a more detailed view of the arrangement of individual microtubules than can be obtained by volume-rendering techniques (compare with Fig. 3B). Stereo pairs are arranged for the cross-eye viewing method. 
Examination of such microtubule cross-sections reveals that reconstructed microtubules are taller than they are wide, indicating artefactual elongation in Z. A hand-traced approximation of the path of the longitudinally orientated microtubule is also shown for reference purposes (Fig. 3C).

The imaging artefacts illustrated in Figs 2 and 3 are more noticeable when volume rendering of an entire data set is attempted. Figure 4 shows stereo-paired views of a complete data set observed along the axis of the microscope (A) and after rotation of $90^{\circ}$ on the $Z$ axis and $75^{\circ}$ on the $X$-axis. Although the visualization of individual microtubules along the original optical axis appears to be satisfactory when viewed along the optical axis (Fig. 4A) when viewed at an angle (Fig. 4B) it is apparent that the resolution in $\mathrm{Z}$ is insufficient to accurately represent the microtubule array in three dimensions.

Figure 5 shows corresponding stereo views of the microtubule array after tracing, application of curve-fitting algorithms and rendering. Because of the difficulty in discriminating closely packed microtubules within the central region of the microtubule array, individual microtubules were traced only as long as they could be clearly detected, and the area surrounding the centrosome is missing from the curve-fit display (asterisks, Fig. 5A). However, in regions where individual microtubules can be clearly detected, comparison of stereo views taken along the optical axis suggests that the curve-fit paths are representative of the course of individual microtubules. The advantages of this approach are clearly visible when data sets viewed from angles other than along the optical axis of the microscope are compared (Figs. 4B and 5B). Although the course of individual microtubules are all but completely obscured when viewed in volume rendered images (Fig. 4B), Bezier curve fit data can be observed at any angle and magnification while retaining clarity and resolution. In this case a single microtubule can clearly be observed to curve upward as it approaches the cell periphery (arrow Fig. 5B), and may penetrate an upwardly extended lamellar protrusion.

To test the accuracy of these methods, we applied the data selection and curve fitting protocols described above to test curves processed using a model of the distortion generated by $z$-dimensional optical sectioning (see Methods). Figure 6(A) shows a test curve upon which has been overlaid the vertical extent of three imaging windows among those used to section the curve. Each window overlaps its neighbours by $10 \%$. Figure 6 (B) shows one of these sections (labelled 2 in Fig. 6A) before (upper panel) and after application of a Z-dimensional smoothing kernel which varies in intensity as a function of distance from the vertical centre of the image (see Methods). The distortion produced is similar to that seen in $\mathrm{XZ}$ sections of biological specimens (compare with Fig. 3). In the process of data acquisition from real specimens, each optical section is represented by a single image. To model this process, each image segment was projected vertically onto an image 1 pixel in height. Both a 'brightest pixel' and an 'average pixel' projection were used, with indistinguishable final results (not shown). The example illustrates the use of a vertical average of pixel intensities. A computationally generated $\mathrm{XZ}$ section is shown through the entire series of 1 pixel high images (Fig. 6C), first without Z-scaling correction (upper panel) and after re-expansion to the original $Z$ dimension (lower panel). The final reconstructed $\mathrm{XZ}$ section resembles the

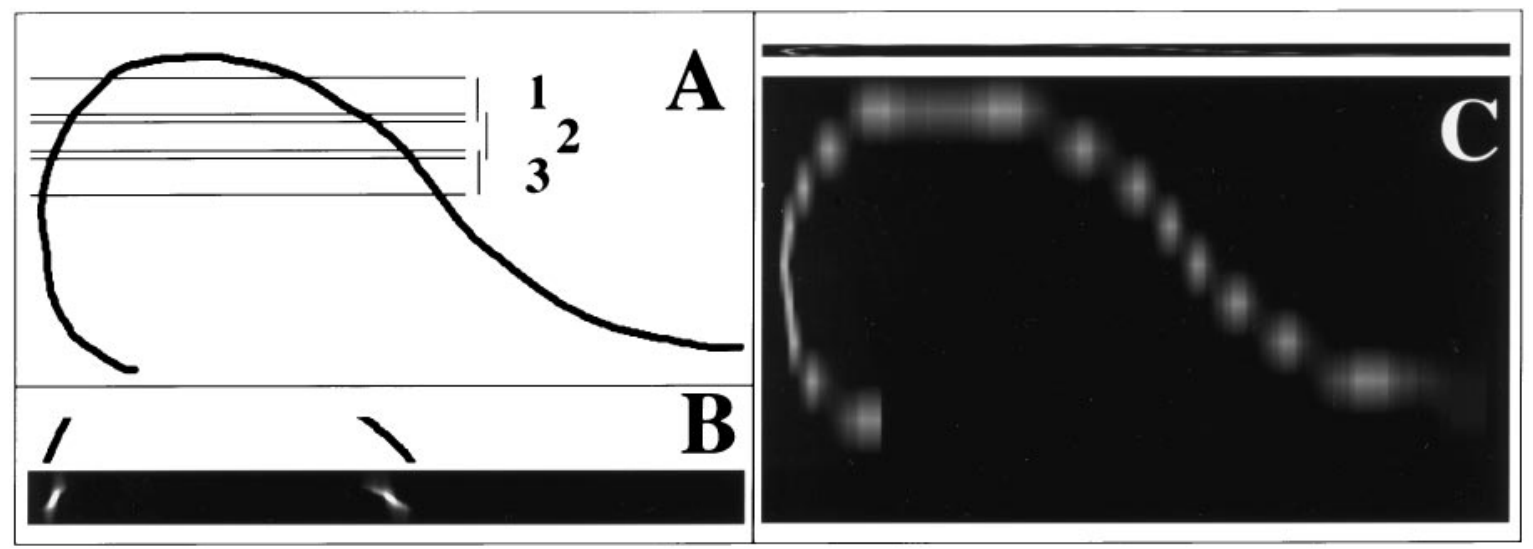

Fig. 6. Modelling of object distortion generated by optical sectioning. (A) Hand-drawn curve modelling a $0 \cdot 2-\mu$ m-thick image of a microtubule within a cell $10 \mu \mathrm{m}$ tall and $40 \mu \mathrm{m}$ in diameter which extends from the central base of the cell over a spherical nucleus and then traverses the upper surface of the cell toward the cell periphery. A pixel $/ \mu \mathrm{m}$ ratio of 20 is assumed. The positions of three overlapping sectioning windows 25 pixels tall are also shown. (B) The central sectioning window in (A) is shown before (upper panel) and after (lower panel) application of a linear z-blurring function. (C) The complete series of sectioning windows is shown after projection of each window onto a single image plane before (upper panel) and after (lower panel) re-establishment of the original Z-dimensions. 


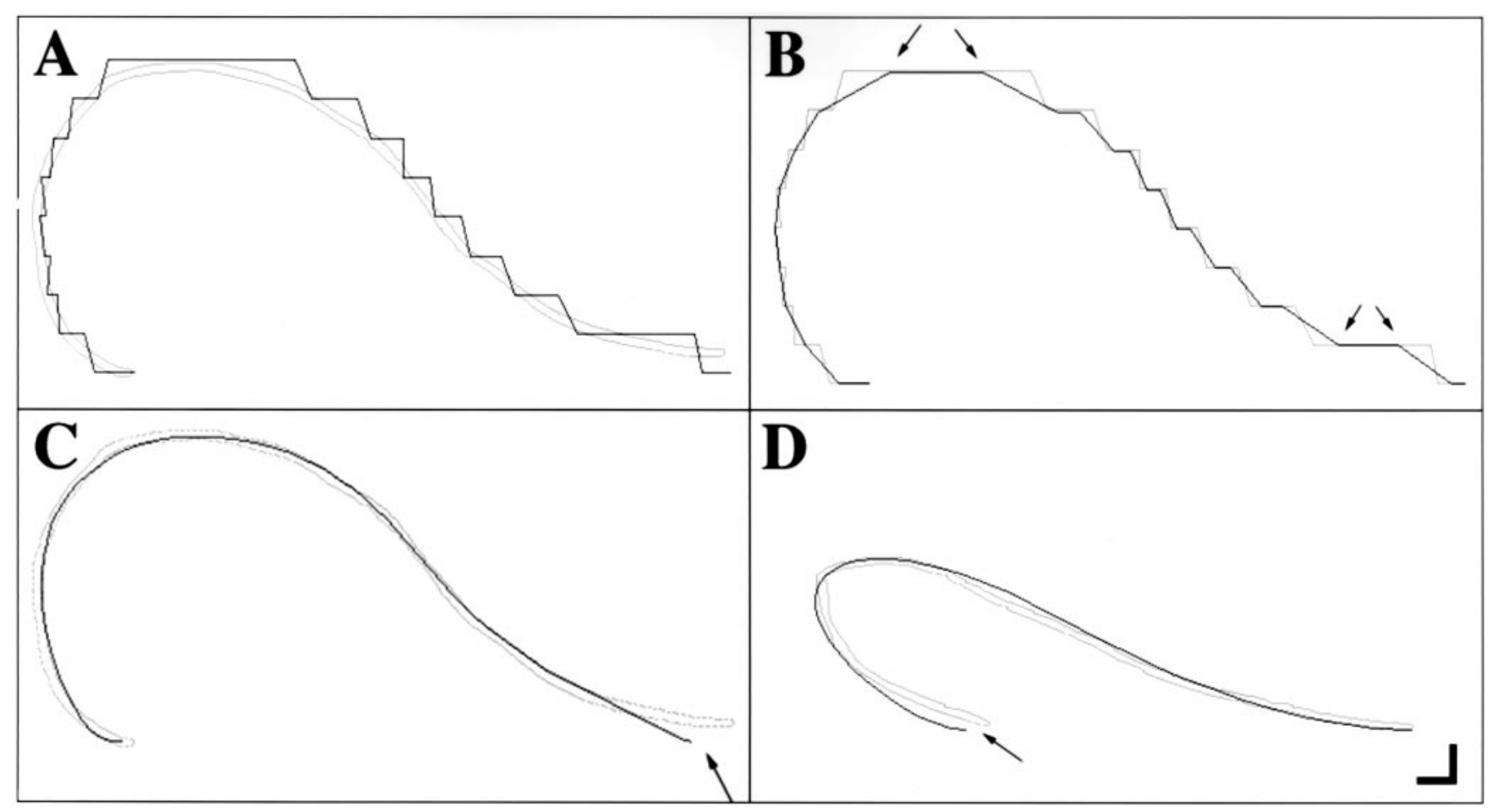

Fig. 7. Comparison of synthesized curves and curves derived from various modelling methods. (A). Original test curve overlaid with a trace generated by connecting data points generated by user-selection from optical section images. (B). The derived trace from panel A is shown overlaid with a path generated through the midpoints of each line segment connecting coplanar user-selected data points. Areas of the curve represented by two linked coplanar line-segments in the original data set are represented by coplanar midpoints here (arrows). (C) A Bezier curve fit to the midpoint data set is shown overlaid onto the original input curve. Overall close correspondence of the Bezier and original curve is evident, although the fit is least accurate at the curve endpoints (arrows). (D). The Bezier fit curve applied to an input curve with a smaller radius of curvature. Bar $=20$ pixels.

original curve in overall geometry, but suffers from a lack of Z-dimensional resolution similar to that seen in volumerendered views of real cytoskeletal filaments (compare with Fig. 4). It is evident that the simultaneous analysis of more than a small number of curves distorted in this manner would be difficult.

Finally, Fig. 7 illustrates the sequence of manipulations during the curve fitting process applied to synthetic curves modified in the manner described above. Figure 7(A) illustrates the outline of an original curve overlaid with a trace generated by linear interpolation between userselected data points using methods described previously (Mathog, 1985). In Fig. 7(B) we show an overlay of a trace through the midpoints of user-defined line segments comprising the trace illustrated in Fig.7(A). It can be observed that several areas of the original curve are represented by at least two segments, and are represented here by adjacent coplanar data points (arrows). The curve generated through the midpoint of the user-selected data more closely resembles that of the original curve than the initial user-selected data set, and comprises fewer data points. However, it is still made up of numerous linear segments, and a simultaneous display of several interwoven traces generated in this manner would probably be difficult to interpret.

Figure 7(C) shows the Bezier curve fit through the midpoint data set overlaid on the outline of the original test curve (Fig. 6A). Comparison of the two curves shows that the deviation of the derived curve from the original is less than the dimension of the sectioning window in all regions. The poorest fit is found at the end-points of the curve (arrows) where the derived curve is constrained through the original data points. Further comparison of the two curves reveals that the poorest fit (right-most endpoint) results where the end of the original curve lies mid-way between two sectioning windows. In Fig. 7(D) we illustrate the application of these techniques to a synthesized test curve with a much smaller radius of curvature, with similar results.

\section{Discussion}

The methods described here use information obtained from optical section images to generate models of filamentous 
structures which can be viewed without loss of resolution from any angle. These methods are particularly suited to situations in which several filamentous objects must be visualized together. Although these methods would probably benefit from application to images in which the axial image resolution is improved using deconvolution techniques, we demonstrate that accurate representations of 3-D filament paths can be generated from optical section images without prior deconvolution. Modelled objects are derived from a small number of user-selected data points, and the methods described here generate useful images of 3-D filament morphology from a smaller number of optical sections than would be required for application of volume rendering approaches at similar resolutions. Thus, these methods can reduce both the amount of time required to obtain volumetric data and the computational resources required to store and visualize the data sets. This approach may therefore be suited to applications, such as the visualization of 3-D structure over time, where a limited number of optical sections can be collected for each volumetric data set. The reduced computation required for the display and analysis of curve fit data as compared to the generation of volume rendered views allows this analysis to be accomplished by inexpensive and readily available desktop computers using commercially available software. Although these methods are particularly suited to the display of unbranched cytoskeletal filaments, they could be adapted to the visualization of a variety of similar biological filaments or synthetic polymers.

To test the validity of our approach, we have applied our reconstruction procedures to test curves which were processed using a model of the z-dimensional distortion produced during optical sectioning. Our results demonstrate that the data selection and rendering methods can produce fit curves which closely resemble the synthesized originals. When the dimensions of these curves are scaled to that appropriate for microscope images, we find that fit curves approximate the input data more closely than the Zdimensional distance between modelled optical sections. The fit curves most poorly approximate the input data at curve ends (see arrows, Fig. 7C), presumably as a consequence of constraining the Bezier curve through the original user-selected endpoints. This leads us to the somewhat surprising observation that the modelled curve points more accurately locate the original curve in the $\mathrm{Z}$ dimension than the original user-selected data set. Our results therefore support the general conclusion that the 3D localization of filamentous objects from optical sections can be improved by approaches recognizing that object elements are part of a whole, rather than a collection of discrete and unconnected voxels.

Comparison of the original and Bezier fit curves in our tests suggest that modelling methods can accurately locate the path of filamentous objects from images containing both
Z-dimensional blurring and out-of-focus contributions from neighbouring image planes. Our model of optical sectioning artefacts incorporates a total $20 \%$ contribution to each image plane from neighbouring images. Although we have not tested the effects of varying the amount of out-of-focus information on the final modelled curves, it seems likely that limiting the out-of-focus contribution of neighbouring planes through the use of reduced confocal pinholes or digital deconvolution techniques will improve the accuracy of the curves generated by our modelling techniques. These improvements may be of particular importance to the accurate localization of filament end-points. However, the accuracy of the current methods in replicating the path of test curves suggests that the curve-fitting strategies described here can tolerate some uncertainty in the localization of line segment end-points which might arise from either noise or inappropriate sampling. This is probably a result of two factors. First, our methods fit the mid-point of each line segment within an optical section, rather than the segment end-points, and uncertainty in the localization of segment endpoints arising as a consequence of noise or out-of-focus blur may be symmetrical around the segment midpoint under many circumstances. Second, the curve-fitting algorithm used here is not constrained through the original data points, with the exception of the two filament end-points. Thus, small errors in the localization of individual line segments have only small effects on the overall shape of the fit curve.

Application of these methods to real images relies upon the accuracy of our modelling of image distortion generated during optical sectioning and the applicability of Bezier curves to representation of the modelled filaments. Our model of Z-dimensional blurring is substantially less complex than that proposed by developers of 3-D deconvolution algorithms (see reviews by Agard et al., 1989; Shaw, 1993) in that it assumes a linear reduction in resolution and signal intensity as a function of distance from the focal plane and ignores the issue of lateral blurring. However, other investigators have noted that the point spread function (a function of the distortion of an optical system produced when imaging a point light source) of a confocal microscope does not show significant lateral spreading (Brakenhoff et al., 1979; Shaw \& Rawlins, 1991). This has led other authors to propose similarly simplified one- (Z) dimensional algorithms for use in the deconvolution of confocal optical sections (Cox \& Sheppard, 1993) and suggests that the strategy for modelling Z-dimensional distortion employed here is a useful approximation of confocal optical sectioning. Indeed, it is possible that linear blurring overestimates the out-of-focus contribution of neighbouring planes to each image. However, our computational model of optical sectioning is intended to represent a 'worst-case' scenario, with the expectation that improvements in optical sectioning resolution by any of a variety of 
methods will allow the generation of still more accurate filament representations.

A second major consideration is the applicability of Bezier curves to modelling of biological specimens. Our implementation of the Bezier curve fitting algorithm fits the entire collection of data points selected for each filament. Such an approach is most appropriate to modelling objects resembling relatively stiff fibres which undergo a small number of smooth curves. Path twists and bends smaller than the Zresolution of the input data will of course not be represented accurately. Evidence obtained at both the electron and light microscope level suggests that Bezier curves are indeed an appropriate model for microtubules under most circumstances. For example, elegant studies conducted by hand tracing microtubules through serial EM sections of mitotic spindles, microtubules (Ding et al., 1993) and microtubule bundles found in neurite extensions (Jacobs \& Stevens, 1986) have shown that microtubules are generally straight structures or undergo only gradual bends. These static images have led some to suggest that microtubules serve as stiffening struts in cells which resist contractile forces (Ingber, 1993; Brown etal., 1996). Impressions of the physical characteristics of microtubules created by static images have been largely confirmed by direct observation of microtubule behaviour in vivo and in vitro (Walker etal., 1988; Cassimeris etal., 1988; Shelden \& Wadsworth, 1993), and in experiments in which the stiffness of microtubules is directly measured by manipulation of microtubule ends in optical traps (Kurachi et al., 1995). In some cases, however, highly stabilized microtubules found within the central region of nondividing cells and those found within dynamic motile structures such as nerve growth cones have been observed to be much more contorted (Schulze \& Kirschner, 1987; Tanaka \& Kirschner, 1991). It is likely that attempting to fit a single Bezier curve through data points derived from such filaments would not result in an accurate three-dimensional picture of their paths. Similarly, the curve-fitting methods used here may not accurately represent extended filaments which undergo numerous bends, such as microtubules within highly elongated nerve fibers. However, several authors have applied Bezier and other curve-fitting algorithms to modelling of curve subregions and devised techniques allowing these segments to be smoothly connected (see discussion by Foley etal., 1990). Future application of such techniques may allow the curve-fitting approaches described here to accurately represent more contorted 3-D filaments.

Finally, several factors limiting the general applicability of the methods described should be addressed. First, these methods rely on user determination and tracing of individual linear objects within image planes. Although hand tracing of image data remains a commonly used technique, this approach is time consuming and limits the number of objects which can be reconstructed and examined. However, a number of investigators have developed programs capable of automated tracking of objects in 2-D or 3-D image series (for examples, see Soll, 1988; McNally, 1995). Although these programs are normally used to track the movement of objects over time, similar automated approaches could be used to link connected object segments, perhaps defined by their proximity and orientation, within a series of optically sectioned images. Secondly, although the approach described above constrains the Bezier curves through the original data at the end-points of each linear object, the path of the object is 'fit' through the intervening $X Y Z$ coordinates and the resulting path points do not generally correspond to the original data points. Although this allows the resulting fit curves to tolerate some error in the localization of individual segment data points, revision of these approaches will be required to represent situations in which the position of branch points and bifurcations must be defined.

\section{Acknowledgments}

This work was supported by grants from the NIH (GM40599) and the Catherine and Weldon Donaghue Medical Research Foundation to D.A.K. and by a postdoctoral fellowship from the Catherine and Weldon Donaghue Medical Research Foundation to E.S.

\section{References}

Agard, D.A., Hiraoka, Y., Shaw, P. \& Sedat, J.W. (1989) Fluorescence microscopy in three dimensions. Meth. Cell Biol. 30, 353-377.

Barillot, C., Gibaud, B., Scarabin, J. \& Coatrieux, J. (1985) 3D reconstruction of cerebral blood vessels. IEEE Comput. Graphics Applications, 5 (12), 13-19.

Brakenhoff, G.J., Blom, P. \& Barends, P. (1979) Confocal scanning light microscopy with high aperture immersion lenses. J. Microsc. 117, 219-232.

Brakenhoff, G.J., Visscher, K. \& Van der Voort, H.T.M. (1989) Size and shape of the confocal spot: Control and relation to 3-D imaging and image processing. The Handbook of Biological Confocal Microscopy (ed. by J. Pawley). IMR Press, Madison WI.

Brown, R.A., Talas, G., Porter, R.A., McGrouther, D.A. \& Eastwood, M. (1996) Balanced mechanical forces and microtubule contribution to fibroblast contraction. J. Cell Physiol. 169, 439-447.

Carrington, W.A., Lynch, R.M., Moore, E.D., Isenberg, G., Fogarty, K.E. \& Fay, F.S. (1995) Superresolution three-dimensional images of fluorescence in cells with minimal light exposure. Science, 268, 1483-1487.

Cassimeris, L., Pryer, N.K. \& Salmon, E.D. (1988) Real-time observations of microtubule dynamic instability in living cells. J. Cell Biol. 107, 2223-2231.

Chasen, S.H. (1975) Geometric Principles and Procedures for Computer Graphics Applications. Prentice-Hall, Englewood Cliffs, NJ. 
Cohen, A.R., Roysam, B. \& Turner, J.N. (1994) Automated tracing and volume measurements of neurons from 3-D confocal fluorescence microscopy data. J. Microsc. 173, 103-114.

Cox, G. \& Sheppard, C. (1993) Effects of image deconvolution on optical sectioning in conventional and confocal microscopes. Bioimaging, 1, 82-95.

Ding, R.K., McDonald, L. \& McIntosh, J.R. (1993) Three-dimensional reconstruction and analysis of mitotic spindles from the yeast Schizosaccharomyces pombe. J. Cell Biol. 120, 141-151.

Foley, J.D., van Dam, A., Feiner, S.K. \& Hughes, J.F. (1990) Computer Graphics Principles and Practice, 2 edn. Systems Programming Series, pp. 471-531. Addison-Wesley, New York.

Frieder, G., Gordon, D. \& Reynolds, R. (1985) Back-to-front display of voxel-based objects. IEEE Comput. Graphics Image Processing, 5, $52-60$.

Fuji, S., Tsukamoto, Y., Kaneda, Y., Fuji, M., Akeno, K., Matsuo, M., Yamasaki, K. \& Ayers, C. (1986) Three dimensional modeling of cerebral arteries from cineangiograms. Proc. 8th Annual Conference of the IEEE Engineering in Medicine and Biology Society, New York, pp. 109-111.

Guilak, F. (1994) Volume and surface area measurement of viable chondrocytes in situ using geometric modeling of serial confocal data. J. Microsc. 173, 245-256.

Ingber, D.E. (1993) Cellular tensegrity: defining new rules of biological design that govern the cytoskeleton. J. Cell Sci. 104, 613-627.

Jacobs, J.R. \& Stevens, J.K. (1986) Experimental modification of PC12 neurite shape with the microtubule-depolymerizing drug nocodazole: a serial electron microscope study of neurite shape control. J. Cell Biol. 103 (3): 907-915.

Kajiya, J. \& Von Herzen, B. (1984) Ray tracing volume densities. Comput. Graphics, 18, 165-174.

Kurachi, M., Hoshi, M. \& Tashiro, H. (1995) Buckling of a single microtubule by optical trapping forces: direct measurment of microtubule rigidity. Cell Motil. Cytoskel. 30, 221-228.

Lorensen, W.E. \& Cline, H.E. (1987) 'Marching Cubes': A high resolution 3D surface construction algorithm. Comput. Graphics, 21, 163-169.

Mathog, D. (1985) Light microscope based analysis of threedimensional structure: application to the study of Drosophila salivary gland nuclei. II. Algorithms for model analysis. J. Microsc. 137, 253-273.
McNally, J. (1995) 3D analysis of cell movement during normal and myosin II null cell morphogenesis in Dictyostelium. Dev. Biol. $167,118-129$.

Pawley, J. (1989) Fundamental limits in confocal microscopy. Handbook of Biological Confocal Microscopy. IMR Press, Madison WI.

Sammak, P.J. \& Borisy, G.G. (1988) Detection of single fluorescent microtubules and methods for determining their dynamics in living cells. Cell Motil. Cytoskel. 10, 237-245.

Schulze, E. \& Kirschner, M. (1987) Dynamic and stable populations of microtubules in cells. J. Cell Biol. 104, 277-288.

Shaw, P.J. (1993) Computer reconstruction in three-dimensional fluorescence microscopy. Electronic Light Microscopy, pp. 211230. Wiley-Liss, Inc., New York.

Shaw, P.J. \& Rawlins, D.J. (1991) The point-spread function of a confocal microscope; its measurement and use in deconvolution of 3-D data. J. Microsc. 163, 151-165.

Shelden, E. \& Knecht, D.A. (1995) Mutants lacking myosin II cannot resist forces generated during multicellular development. J. Cell Sci. 108, 1105-1115.

Shelden, E. \& Wadsworth, P. (1993) Observation and quantification of individual microtubule behavior in vivo: microtubule dynamics are cell-type specific. J. Cell Biol. 120, 935-945.

Soll, D.R. (1988) 'DMS,' a computer-assisted system for quantitating motility, the dynamics of cytoplasmic flow, and pseudopod formation: its application to Dictyostelium chemotaxis. Cell Motil. Cytoskel. 10, 91-106.

Tanaka, E.M. \& Kirschner, M.W. (1991) Microtubule behavior in the growth cones of living neurons during axon elongation. $J$. Cell Biol. 115, 345-363.

Taylor, D.L. \& Salmon, E.D. (1989) Basic fluorescence microscopy. Methods Cell Biol. 29, 207-237.

Walker, R.A., O'Brien, E.T., Pryer, N.K., Soboeiro, M.F., Voter, W.A., Erickson, H.P. \& Salmon, E.D. (1988) Dynamic instability of individual microtubules analyzed by video light microscopy. $J$. Cell Biol. 107, 1437-1448.

Wilson, T. (1990) The role of the pinhole in confocal imaging systems. Handbook of Biological Confocal Microscopy, 2nd edn (ed. by J. B. Pawley), pp. 113-126. Plenum Press, New York. 